\title{
Stochastic resonance in a model of opinion formation on small-world networks
}

\author{
Marcelo Kuperman ${ }^{1,}$ and Damián Zanette ${ }^{1,2, \text { f }}$ \\ ${ }^{1}$ Centro Atómico Bariloche and Instituto Balseiro, 8400 S. C. de Bariloche, Argentina \\ ${ }^{2}$ Consejo Nacional de Investigaciones Científicas y Técnicas, Argentina
}

(Dated: October 25, 2018)

\begin{abstract}
We analyze the phenomenon of stochastic resonance in an Ising-like system on a smallworld network. The system, which is subject to the combined action of noise and an external modulation, can be interpreted as a stylized model of opinion formation by imitation under the effects of a "fashion wave". Both the amplitude threshold for the detection of the external modulation and the width of the stochastic-resonance peak show considerable variation as the randomness of the underlying small-world network is changed.

PACS numbers: 87.23.Ge, 89.65.-s, 05.40.-a
\end{abstract}

\section{INTRODUCTION}

Many mathematical models of social processes, inspired by analogies with physical systems, have recently been formulated in order to describe a wide spectrum of phenomena [1]. Economic and financial processes, disease spreading and information propagation, and evolution of social structures, among others, have been analyzed along such lines. Weidlich [1] has proposed a model of public opinion formation which, in the simplest variant, considers that the individuals can adopt two different opinions. The opinion of a given individual may be influenced by that of the neighbors, making it to change with a certain probability. In the present work, we analyze a similar model of social influence and opinion formation. In contrast with previous studies of this class of models, however, we are interested at taking into account the network of social interactions. Specifically, we model the underlying social structure of the population as a small-world network. Small-world networks [2] incorporate two main features of real social interactions. First, they are highly clustered, which means that any two neighbors of a given site have a relatively large probability of being in turn mutual neighbors. Second, the mean number of intermediaries between any two sites is quite small, and increases very slowly as the total number of sites grows [3]. This is precisely the small-world property, originally discussed by Milgram as a typical feature of social communities and relationships 鿉. Smallworld networks can be considered as partially disordered structures. The construction procedure makes it possible to control the degree of disorder of a given network, ranging from ordered lattices to completely disordered graphs. Among many other applications, these networks have been used as a model of social structures in the study of disease [2, 5, 6, 7, 8] and rumor propagation [9.

As pointed out in the next section, our model is an Ising-like system whose dynamics is driven by the majority rule. Equilibrium properties of Ising systems on small-world networks have been studied in detail. It has been shown that, in asymptotically large systems, even an infinitesimal amount of disorder on a one-dimensional Ising lattice is sufficient for the system to undergo a ferromagnetic phase transition as the temperature varies [3]. Here, instead, we consider the case where the system is maintained out of equilibrium by an external modulation. In the frame of the problem of opinion formation, this modulation plays the role of a periodic "fashion" wave, as actually observed in certain real situations [10]. The combination of this modulation with noise - which, in equilibrium, would play the role of the temperatureleads naturally to the consideration of stochastic resonance [11. In the phenomenon of stochastic resonance, an enhancement of the response of a system to an external modulation is obtained by an adequate choice of the noise level. This effect has attracted great interest due to both its potential technological applications and its connection with biological detection mechanisms. The range of possible applications in science includes paleoclimatology [12], electronic circuits 13, 14], lasers 115, 16, chemical 17 and biological systems 18, 19. Stochastic resonance in Weidlich's model of opinion formation has already been studied [10]. In the present work, we analyze this phenomenon in a closely related model of social imitation, incorporating a small-world network as the underlying structure of social interactions.

\section{THE MODEL}

As advanced in the Introduction, we consider an Isinglike model on a network with a controlled degree of disorder, namely, a small-world network. The network is constructed as follows [2]. We start with an $N$-node one-dimensional ordered lattice with periodic boundary conditions, where each node is linked to its $2 K$ nearest neighbors ( $K$ clockwise and $K$ counterclockwise). The network is then disordered, rewiring each of the $K$ clockwise connections of each node $i$ to a randomly chosen node $j$, with probability $p$. Double and multiple connections are forbidden, and realizations where the network becomes disconnected are discarded. The final degree of 


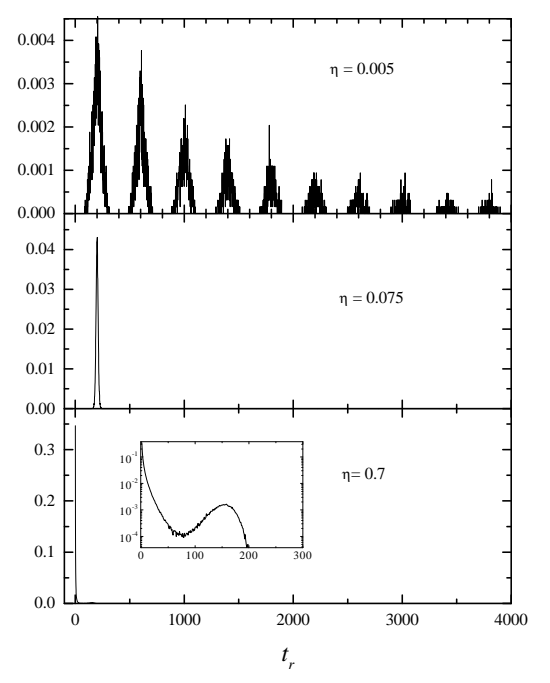

FIG. 1: Normalized histograms of the residence time $t_{r}$, for $p=0.8$. Three different noise intensities $\eta$ are considered. Each histogram has been constructed recording the residence times over an interval of $8 \times 10^{5}$ time units, with a subsequent average over 20 realizations of the small-world network and the initial condition. The insert in the third plot shows the same data with logarithmic scale in the vertical axis.

disorder, or randomness, is determined by the probability $p$. As a result of the disordering process, some shortcuts between otherwise distant regions are created. Note that, independently of the value of $p$, the average number of links per site is always $2 K$.

Each site of the small-world network is occupied by an individual with two possible states, $\mu_{i}= \pm 1$. At each time step, an individual $i$ is selected at random from the whole population, and the following three evolution rules are successively applied to its state $\mu_{i}$. First, $\mu_{i}$ is changed according to the majority rule, i.e.

$$
\mu_{i} \rightarrow \operatorname{sign} \sum_{j\{i\}} \mu_{j}
$$

where the sum runs over the neighborhood of site $i$. If the sum equals zero, $\mu_{i}$ is left unchanged. Second, we apply the external modulation. The effect of this modulation is prescribed by a harmonic function of time, $f(t)=A \cos (2 \pi t / \tau)$, with $0<A<1$. Namely, the state of individual $i$ changes according to

$$
\mu_{i} \rightarrow \operatorname{sign} f(t)
$$

with probability $\Pi_{r}(t)=|f(t)|$. Finally, noise is applied as follows. With constant probability $\Pi_{n}$ the state $\mu_{i}$ is left unchanged. With the complementary probability $1-\Pi_{n}, \mu_{i}$ is assigned one of the two possible values \pm 1 , chosen at random with probability $1 / 2$. We call $\eta=$ $1-\Pi_{n}$ the noise intensity.

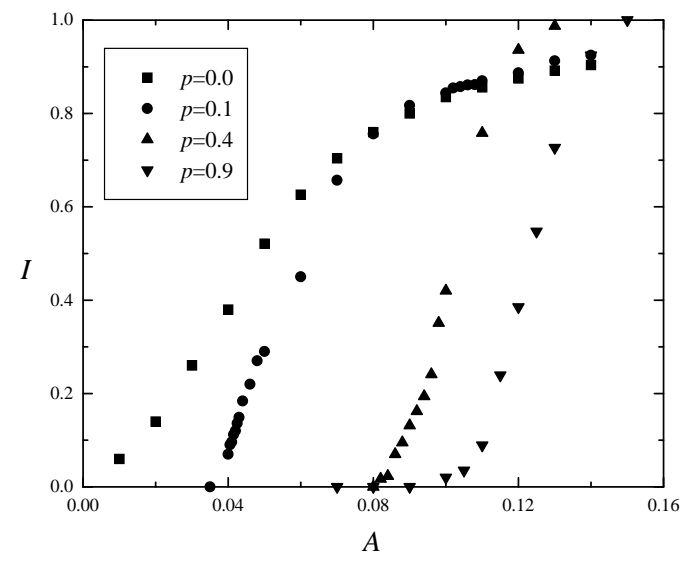

FIG. 2: Response $I$ as a function of the modulation amplitude $A$, for $\eta=0.1$, and four values of the randomness $p$.

In the absence of modulation, at low noise levels, and for moderate and large randomness $p$, our population evolves towards an almost homogeneous state, where $\mu_{i}$ is the same at practically all sites. The average of $\mu_{i}$ over the population, $\bar{\mu}=N^{-1} \sum_{i=1}^{n} \mu_{i}$, is close to \pm 1 . Due to the effect of noise, occasional transitions between states with opposite signs are observed. For sufficiently small $p$, on the other hand, $\bar{\mu}$ fluctuates around zero with relatively small amplitude. These two regimes correspond, respectively, to the ferromagnetic and paramagnetic phases predicted for equilibrium small-world Ising systems [3]. In our simulations, we will mainly consider randomness and noise levels for which the nonmodulated system is in the ferromagnetic-like phase. All the cases with $p>0$ will in fact correspond to that phase. In this situation, the noiseless version of our system exhibits bistability. The paramagnetic-like phase will only be encountered in the special case $p=0$.

The effect of the external modulation on the ferromagnetic-like phase consists of a periodic modification in the transition probability between the two states with $\bar{\mu} \approx \pm 1$. In the paramagnetic-like phase, on the other hand, $\bar{\mu}$ oscillates around zero at the same frequency as the modulation. In both cases, of course, the modulation is combined with the random fluctuations of noise. It is this nontrivial interplay, which may result in the enhancement of the system response, that we focus on in our numerical simulations.

\section{NUMERICAL RESULTS}

We have performed extensive numerical simulations of the model presented in the previous section, considering small-world networks of $N=10^{3}$ nodes with $K=3$. The time interval assigned to an evolution step is $\Delta t=N^{-1}$ 


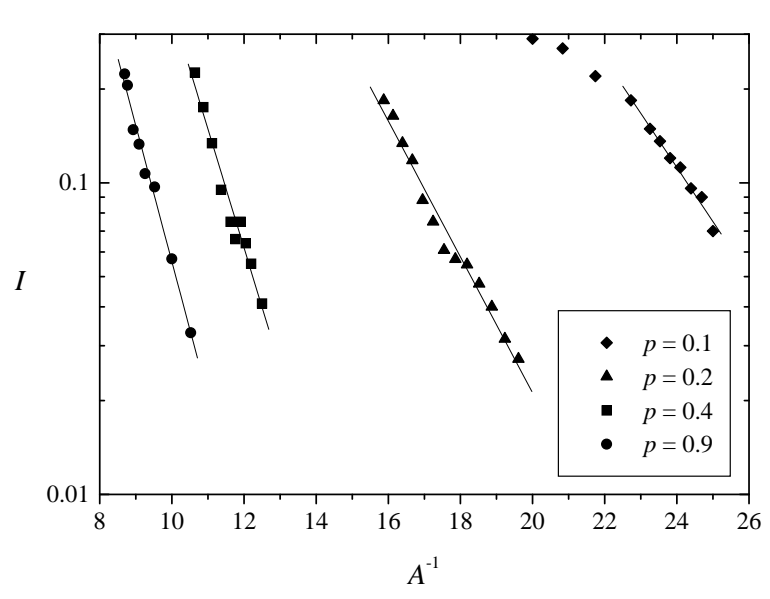

FIG. 3: The response $I$, in logarithmic scale, as a function of the inverse modulation amplitude, $A^{-1}$, for four values of $p$. Straight lines are least-square fittings to the linear regime of each data set.

so that, on the average, the state of each site is updated once during each time unit. Each realization starts with the generation of the random network and the random initialization of the state of all the elements. The evolution is analyzed after a transient of $10^{5}$ time units, during which the system is found to reach a steady large-time behavior. In particular, we record the residence times $t_{r}$, i. e. the intervals between two consecutive changes of sign in the average state $\bar{\mu}$. Note that the external modulation is expected to induce changes in the sign of $\bar{\mu}$ with a typical period close to $\tau / 2$. The values of $t_{r}$ are used to construct a histogram, showing the frequency of occurrence of each residence time. Throughout all the calculations we considered $t_{r}=400$.

Figure 1 illustrates this histogram for fixed modulation and small-world randomness, and three levels of noise. For low noise, the histogram shows a peak at $t_{r}=\tau / 2$, as expected, but several odd harmonics, $t_{r}=k \tau / 2$ with $k=3,5,7, \ldots$, are also observed. These harmonics correspond to residence intervals during which the modulation fails, one or more times, at inducing the transition. At intermediate noise levels, corresponding to the resonance intensity, only the pick around $t_{r}=\tau / 2$ is important. For higher noise levels the transitions are disordered and highly frequent, so that the residence times are usually very short and the histogram shows a peak near $t_{r}=0$. We stress that the analysis of these patterns has been employed as a quantitative description of stochastic resonance in other systems [11, 20], as an alternative to the study of the signal-to-noise ratio [11]. In fact, a measure of the sensitivity to the external modulation is given by the area $I$ under the peak around $\tau / 2$ in the (normalized) histogram. Below, we refer to the area $I$ as the response of the system.

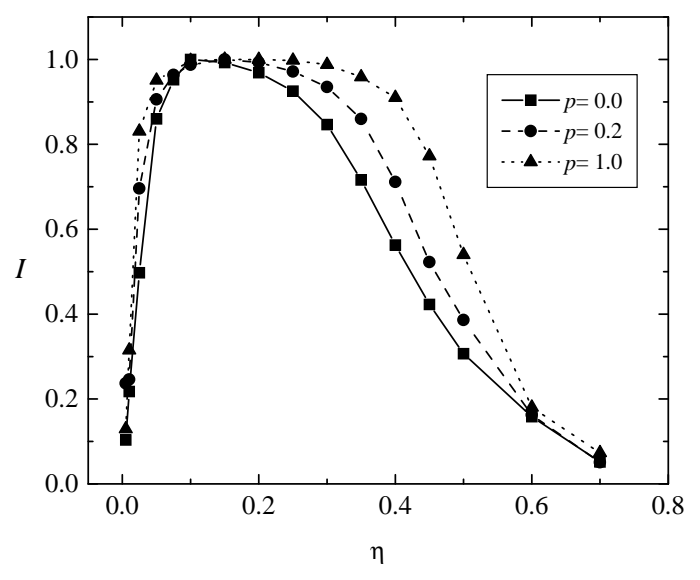

FIG. 4: The response $I$ as a function of the noise intensity $\eta$, for three values of the small-world randomness $p$. Lines joining dots are drawn as a guide to the eye.

First, we analyze the behavior of our system as the modulation amplitude $A$ is varied. Fixing the intensity of noise $\eta$ we observe, for $p>0$, the existence of a threshold in $A$ below which the system is not able to respond to the modulation. We find that, as the randomness $p$ increases, the threshold amplitude grows as well. Figure 2 shows the response $I$ for a noise intensity $\eta=0.1$ as a function of $A$, for several values of $p$. The nature of this threshold phenomenon is clarified in Fig. 3, where we plot $I$ in logarithmic scale as a function of the inverse amplitude for three values of $p$. The approximately linear dependence for small $I$ suggests the functional form

$$
I(A)=I_{0} \exp \left(-A_{0} / A\right)
$$

This form is reminiscent of Kramers formula for the transition frequency $\omega$ between equilibrium states under the action of thermal noise $T, \omega \propto \exp \left(-T_{0} / T\right)$, where $T_{0}$ is related to the potential barrier separating the equilibria [21]. In our case, for a fixed noise level, the role of temperature - as the factor that induces the transitionis played by the external modulation. Exploiting this analogy, our model can be thought of, at the macroscopic level, as a system subject to an effective bistable potential, with a barrier proportional to the slope $A_{0}$. It is apparent from Fig. 3 that the slope depends on the small-world randomness. As $p$ grows from 0.1 to 0.9 , the slope increases by a factor of about 2.5, indicating that the minima in the effective potential become deeper for larger randomness.

Taking into account the results shown in Figs. 2 and 3, in our study of dependence of the response $I$ on the noise intensity $\eta$ we fix $A=0.2$ which, for the values of $p$ and $\eta$ considered in our simulations, is always above the detection threshold. In Fig. A, we show $I$ as a function of eta for several values of $p$. The existence of a resonance 


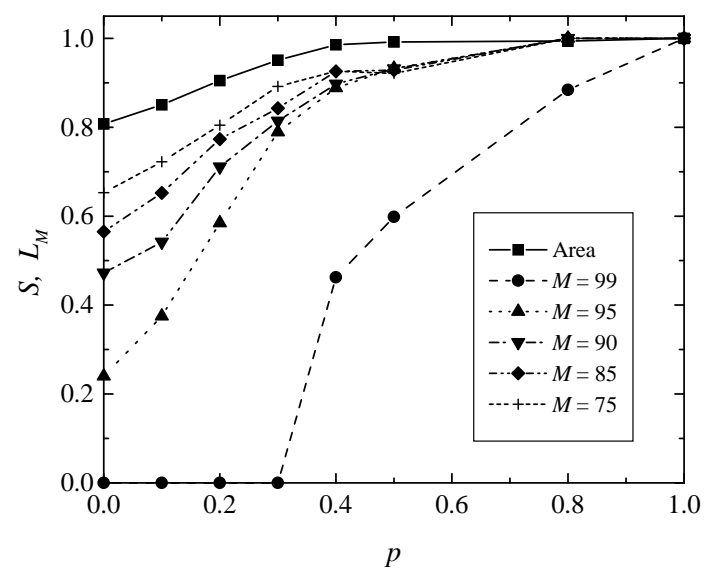

FIG. 5: Area $S$ under the stochastic-resonance curves (Fig. 44) and lengths $L_{M}$ for several values of $M$ (see main text), as a function of the randomness $p$. Lines joining dots are drawn as a guide to the eye.

as the noise intensity is varied is apparent. At the same time, an effect related to the variation of the randomness $p$ is observed. Namely, the higher is $p$, the broadest is the resonant interval in the eta. In other words, accurate tuning of the noise level in order to enhance the response is less important in more disordered networks.

Figure 5 presents a quantitative characterization of the broadening of the resonance peaks as $p$ grows. First, we show the area $S$ of the peaks as a function of $p$. Moreover, we show the length $L_{M}$ of the intervals of noise intensity where the response $I$ is above the level $I_{M}=0.01 M$, for several values of $M$.

\section{CONCLUSION}

We have presented an Ising-like model of opinion formation by imitation on a small-world network, subject to the action of an external modulation and noise. The study is focused on the phenomenon of stochastic resonance, which underlies the macroscopic behavior of this system. The main control parameters in our analysis are the amplitude $A$ of the external modulation, the intensity of noise $\eta$, and the small-world randomness $p$. The first aspect studied here is the existence of a threshold in the amplitude of the external signal, below which the system is not able to detect the modulation. This threshold is higher as the disorder of the network increases, and disappears for $p=0$. This fact suggests that the existence of a threshold in $A$ is directly related to the bistable nature of the effective potential which acts on the system in its ferromagnetic-like phase, i. e. for moderate and large randomness. It is interesting to mention that the increase of the threshold amplitude with the randomness, which amounts to a growth in the effective potential barrier, has a well-known correlate in social imitation processes subject to the action of noise [22]. The resistance to collective changes of opinion grows drastically when long-range social interactions are allowed. A population restricted to local interactions, instead, is susceptible to global opinion transitions by diffusive-like propagation.

As expected, the analysis of the response of the system as a function of the noise intensity $\eta$ - provided that the amplitude $A$ is above the detection threshold-reveals the occurrence of a resonance phenomenon. Though the response of the system at the resonance is practically independent of the randomness $p$, an enhancement of the phenomenon is apparent from the broadening of the resonance curves as a function of $\eta$ (Figs. 4 and 5 ). The convenience of broadening the stochastic-resonance peak to avoid noise tuning has been discussed in connection with a variety of systems [20, 23, 24].

The existence of stochastic resonance in a model of opinion formation yields the appealing implication that there is an optimal noise level for a population to respond to an external "fashion" modulation [10]. Lower noise intensities lead to the dominance of the majority's opinion, irrespectively of the external influences, while sufficiently stronger fluctuations prevent the formation of a definite collective opinion. We have here shown that, in this phenomenon, the underlying structure of social interactions plays a role, allowing for a looser tuning of the resonance noise intensity as the disorder grows.

Let us finally mention that we have tested the robustness of these results by studying a few variations of our model. For instance, we have considered the case where the external modulation acts only when it is not possible to define an individual's state by the majority rule, i. e. when the sum in Eq. (11) equals zero. In this case, the individual adopts the state dictated by the "fashion" wave with probability $\Pi_{r}(t)$, defined in Sect. II, or preserves the previous state with the complementary probability. In all cases we got consistent results, qualitatively similar to those presented here. Though our model is an oversimplified caricature of any real process, the results illustrate the complex interplay between fluctuations, external influences, and interaction structure that is expected to take place in actual societies.

The authors thank H. S. Wio for fruitful suggestions. MK thanks Fundación Antorchas for financial support.

* Electronic address: kuperman@cab.cnea.gov.ar

$\dagger$ Electronic address: zanette@cab.cnea.gov.ar

[1] W. Weidlich. Phys. Rep. 204, 1 (1991).

[2] D. J. Watts and S. H. Strogatz, Nature 393, 440 (1998).

[3] A. Barrat and M. Weigt, Eur. Phys. J. B 13 (2000) 547560.

[4] S. Milgram, Psychol. Today 2, 60 (1967). 
[5] M. Kuperman and G. Abramson, Phys. Rev. Lett. 56, 1906 (2001).

[6] C. Moore and M. E. J. Newman, Phys. Rev. E 60 (1999) 5678.

[7] R. Pastor-Satorras and A. Vespignani, Phys. Rev. E 63, 066117 (2001).

[8] N. Zekri and J. P. Clerc, Phys. Rev. E 64, 056115 (2001).

[9] D. H. Zanette, Phys. Rev. E 64 050901(R) (2001).

[10] P. Babinec, Phys. Lett. A, 225, 179 (1997).

[11] L. Gammaitoni, P. Hänggi, P. Jung and F. Marchesoni, Rev. Mod. Phys. 70223 (1988).

[12] R. Benzi, A. Sutera and A. Vulpiani, J. Phys. A 14, L453 (1981).

[13] S. Fauve and F. Heslot, Phys. Lett. A 97, 5 (1983).

[14] R. N. Mantegna and B. Spagnolo, Phys. Rev. E 49, R1792 (1994).

[15] J.M. Iannelli, A. Yariv, T.R. Chen and Y.H. Zhuang, Appl. Phys. Lett. 65, 1983 (1994).
[16] A. Simon and A. Libchaber, Phys. Rev. Lett. 69, 3375 (1992).

[17] V. Petrov, Q. Ouyang and H. L. Swinney, Nature 388, 655 (1997).

[18] J. K. Douglas, L. Wilkens, E. Pantazelou and F. Moss, Nature 365, 337 (1993).

[19] S. M. Bezrukov and I. Vodyanoy, Nature 378, 362 (1995).

[20] F. Castro, M. Kuperman, M. Fuentes and H. Wio, Phys. Rev. E 64, 0511058 (2001).

[21] C. W. Gardiner, Handbook of Stochastic Methods for Physics, Chemistry and the Natural sciences (Berlin, Springer, 1985).

[22] H. Peyton Young, Individual Strategy and Social Structure (Princepton University Press, Princeton, 1998).

[23] S. Bouzat and H. S. Wio, Phys. Rev. E 59, 5142 (1999).

[24] C. J. Tessone, H.S. Wio and P.Hänggi, Phys. Rev. E 62, 4623 (2000). 